\title{
S. Haematobium Infection and Chemotherapy-Induced Changes in Interleukin-6 and Acute Phase Proteins Associated with Inflammation in School Children in a Schistosomiasis-Endemic Area
}

\author{
Tawanda Chisango ${ }^{1 *}$, Arthur Vengesai ${ }^{2}$, Agness Farai Nhidza ${ }^{1}$, Bongiwe Ndlovu ${ }^{1}$, Danai Zhou ${ }^{3}$, Edson Sibanda ${ }^{4}$ and Takafira Mduluza ${ }^{1,2}$ \\ ${ }^{1}$ School of Laboratory Medicine and Medical Sciences, College of Health Sciences, University of KwaZulu-Natal, KwaZulu-Natal, South Africa \\ ${ }^{2}$ Biochemistry Department, University of Zimbabwe, Harare, Zimbabwe \\ ${ }^{3}$ Medical Laboratory Sciences, College of Health Sciences, University of Zimbabwe, Harare, Zimbabwe \\ ${ }^{4}$ Scientific and Industrial Research and Development Centre, 1574 Alpes Road, Box 6640, Harare, Zimbabwe
}

"Corresponding author: Tawanda Chisango, School of Laboratory Medicine and Medical Sciences, College of Health Sciences, University of KwaZulu-Natal, KwaZuluNatal, South Africa, Tel: +263772731322; E-mail: chisangotawanda@yahoo.com

Received date: March 05, 2018; Accepted date: June 01, 2018; Published date: June 08, 2018

Copyright: $\odot 2018$ Chisango T, et al. This is an open access article distributed under the terms of the Creative Commons Attribution License, which permits unrestricted use, distribution and reproduction in any medium, provided the original author and source are credited.

\begin{abstract}
Objective: There is an increased risk of cases of direct and indirect morbidities as a result of stimulation of tissue-destructive inflammation caused by Schistosoma haematobium infection, hence the need to determine the levels of inflammatory markers in Schistosoma haematobium infected children and also determine the effect of repeated annual mass treatment on levels of interleukin- 6 and acute phase proteins.
\end{abstract}

Methodology: Urine specimens from 212 school children were collected and examined to determine prevalence of Schistosoma haematobium at baseline and 2 years following annual rounds of praziquantel treatment. Levels of 4 acute phase proteins were measured from serum samples from the participants using the magnetic bead-based immuno-assays at baseline and 2 years following praziquantel treatment. Sandwich enzyme-linked immunosorbent assay was used to determine levels of interleukin-6.

Results: The overall pre-treatment prevalence of Schistosoma haematobium infection was $23.1 \%$ at baseline and $0.47 \%$ after 2 years of annual treatments. Schistosoma haematobium infected children had marginally higher levels of procalcitonin and tissue plasminogen activator before treatment though the difference of all three was not significant $p>0.05$ using Mann-Whitney non-parametric $U$ test. Levels of ferritin and fibrinogen were lower in Schistosoma haematobium infected children before treatment, however the difference was also not significant $p>0.05$ using Mann-Whitney test. There was no association between infection status or interleukin- 6 and the levels acute phase proteins $p>0.05$ for all acute phase proteins using the Mann-Whitney $U$ test.

Discussion and Conclusion: Findings from this study suggest no bearing of Schistosoma haematobium infection status on level of acute phase proteins before and after annual treatment with praziquantel. The extent of inflammation cannot be determined using ferritin, tissue plasminogen activator and fibrinogen. Levels of interleukin-6 did not have any bearing on levels of acute phase proteins. There is a need to explore other acute phase proteins as inflammatory markers in Schistosoma haematobium infection.

Keywords: Schistosoma haematobium; Procalcitonin; Tissue plasminogen activator; Ferritin; Interleukin-6

\section{Abbreviations:}

TPA: Tissue Plasminogen Activator;

WHO: World Health Organization;

MDA: Mass Drug Administration;

PZQ: Praziquantel;

APPs: Acute Phase Proteins

\section{Introduction}

Schistosomiasis is a water-borne parasitic disease with a global disease burden calculated at 24-56 million disability-adjusted life-years lost [1]. Chronic inflammation subsequent to infection with $S$. mansoni and $S$. japonicum, and $S$. haematobium appears to be the major source of burden in individuals with shistosomiasis. Subtle morbidities such as anaemia, growth deficiencies, physical fatigue and diminished cognitive development occur as a result of the inflammation [2-6]. There is an increased risk of direct and indirect cases of morbidities as a result of stimulation of tissue-destructive inflammatory and granulomatous reactions from $S$. haematobium infection. In children of school going age infected with $S$. haematobium anaemia associated with chronic inflammation is worsened by blood loss seen as gross and micro haematuria. In addition to this example of direct morbidity, the Schistosoma-infected host can be indirectly predisposed to greater susceptibility to other pathogens. For example, there is an increased risk to HIV acquisition in individuals with friable sandy patches that are common in female genital schistosomiasis caused by $S$. haematobium infections [5-7]. 
In the control of parasitic diseases, immune responses and consequently the inflammatory processes are meant to eliminate the harmful agent and restrict tissue damage but in some situations it may end up triggering pathological repercussions that can also affect injury and illness itself. In some instances inflammation can persevere even after the harmful agent has been eliminated, giving rise to chronic inflammation [8]. It is evident that immune responses and cytokine responses generated during the various stages of the life cycle of the parasite account for most of the morbidities culminating in chronic inflammation against schistosome antigens that are released from eggs trapped in tissues $[9,10]$. The location and number of eggs lodged in the tissues initially determine the magnitude of inflammation and with time the pathology associated with fibrosis and organ damage [11]. In $S$. haematobium endemic areas, children are exposed to the parasite through water related activities such as swimming and those infected continuously have eggs being deposited in their tissues and consequently the immunopathological reactions against these eggs trapped leads to an increase in acute phase proteins and inflammation. When the agent triggering inflammation occurs repeatedly the acute phase response and may be continuously activated and become chronic as the case in $S$. haematobium-endemic areas where there is constant exposure of worms and egg burden. Immune reactions to Schistosoma eggs trapped in the tissues in chronically infected cases ultimately result in inflammation in affected tissues followed by granuloma formation [11].

Praziquantel has become the drug of choice in the treatment of schistosomiasis and it is dispensed through mass drug administration programmes around Africa. The drug is effective against all schistosome species with minimal detrimental effects to the host and it is also effective against other trematode and cestode infections [12]. The mechanism of action is not yet fully understood though there is evidence suggesting that it increases the permeability of schistosome membranes to calcium thereby promoting tegument damage and worm paralysis [13]. The dying parasites are then removed from the host and destroyed by circulating immune cells. There is unequivocal evidence that praziquantel treatment minimizes worm burden thereby reducing the amount of deposited eggs culminating in an overall decline in inflammation related to the immunopathological reactions to the eggs and therefore praziquantel should be able to reduce chronic inflammation especially if administered regularly.

Knowledge of inflammation markers is necessary in assessing and identifying children who are likely to develop chronic pathologies later on in life. Chronic inflammation can be seen as a continuous series of distinct and consistent inflammatory stimuli. In such conditions, increased serum concentrations of acute phase proteins and proinflammatory cytokines are generally observed [14].

Acute phase proteins are a group of plasma proteins derived primarily from the liver and they are involved inhibition of infection, mediating systemic effects like fever, leucocytosis, increased cortisol, decreased serum iron, and many others [15]. The extent of inflammatory tissue damage as well as diagnostic and prognostic information in some human diseases can be resolved by measurement of acute phase proteins [16,17]. Bacterial infections [18], neoplasia, [19] and inflammatory bowel diseases (IBD) have employed measurements of acute phase proteins in their prognosis [20]. However there is inadequate information regarding the use of acute phase proteins in parasitic infections like in human Schistosoma infection $[21,22]$. This is unusual considering the fact that parasitic infections elicit considerable inflammation.
Circulating microbial products are well known inducers of acute phase proteins [23], thus, in $S$. haematobium infected children the microbial products from the natural death of the schisitosomes and those released following praziquantel treatment might induce overproduction of acute phase proteins. In this study, fibrinogen, ferritin and tissue plasminogen activator are going to be evaluated before and after treatment with praziquantel. Acute phase proteins have varying half-lives, rising and falling at different times, which limit the use of only one biomarker in determining inflammation. Thus the simultaneous measurements could be most effective in identifying individuals prone to chronic inflammation.

Traditional biomarkers such as C-reactive protein, serum amyloid A and haptoglobulin have produced conflicting results in terms of correlation between inflammation and schistosomiasis and insufficiently sensitive or specific enough to guide treatment decisions in infectious diseases. Some studies have reported a direct relationship between schistosomiasis and presence of the acute phase proteins [2426] whilst other researchers have found no link between the two. To our knowledge the combination of tissue plasminogen activator, fibrinogen, ferritin and procalcitonin has not been evaluated in individuals living in schistosomiasis endemic areas. Recently, there has been interest in the potential use of procalcitonin and ferritin as inflammatory markers in various infectious diseases as a result of the increase of both retrospective and prospective studies that consistently have documented elevated serum concentrations of procalcitonin and ferritin in various parasitic infection.

Acute phase proteins are released as a result of the action of cytokines such as IL-1, IL- 6 , and TNF- $\alpha$ produced by T-lymphocytes, macrophages, monocytes, endothelial cells, and fibroblasts at the site of inflammatory lesions. Although it is evident that a number of proinflammatory and anti-inflammatory cytokines are involved in the inflammatory response [27], available data indicates that IL-6 is the supreme stimulator $[21,28]$. Acute inflammation turns into chronic inflammation if the activity of IL-6 perseveres. However, in knockout mice incapable of expressing IL-6, the role of IL- 6 in triggering the production of acute-phase proteins depends on the nature or site of the inflammatory stimulus; the response is largely inhibited in IL-6 knockout mice injected with turpentine but is normal when bacterial lipo-polysaccharide is the inflammatory stimulus [22]. Unrestrained and sustained action of cytokines is potentially harmful. Anti-cytokine therapies are thus useful in light of the role of proinflammatory cytokines in inflammation-related pathologies and this may have far reaching consequences in schistosomiasis vaccine development which has been elusive [29].

Identifying individuals at an early stage who are vulnerable to inflammation allows better prognosis and prevents the use of invasive, costly and time consuming procedures to determine those suffering from pathological complications of chronic inflammation conditions. It has been demonstrated that biomarkers $\mathrm{C}$-reactive protein and faecal calprotectin can be used to evaluate disease status in patients with inflammatory bowel disease (IBD) though endoscopic evaluation which is expensive and invasive is the gold standard [30]. The goal of disease monitoring is to identify individuals at risk in order to treat earlier.

Although perpetual and continuous inflammation is presumed to be a hallmark of schistosomiasis, very few studies have actually examined the acute phase proteins simultaneously with the proinflammatory cytokine IL-6 in $S$. haematobium infections. In this study $S$. haematobium infections was first determined then determined ferritin, 
Citation: $\quad$ Chisango T, Vengesai A, Nhidza AF, Ndlovu B, Zhou D, et al. (2018) S. Haematobium Infection and Chemotherapy-Induced Changes in Interleukin-6 and Acute Phase Proteins Associated with Inflammation in School Children in a Schistosomiasis-Endemic Area. J Trop Dis 6: 266. doi:10.4172/2329-891X.1000266

Page 3 of 7

fibrinogen, tissue plasminogen activator, procalcitonin and IL-6 at baseline to assess inflammation in both $S$. haematobium-infected and $S$. haematobium uninfected school children. Secondly we established if repeated rounds of praziquantel treatment for 2 years results in changes in the acute phase proteins and IL-6.

\section{Methodology}

\section{Study population and study area}

The study was carried out in Bandanyenje primary school located in a schistosomiasis endemic setting in the Manicaland Province of Zimbabwe. It is located approximately $217 \mathrm{~km}$ south of the capital city Harare with latitude and longitude of $7^{\circ} 1^{\prime} \mathrm{N} 38^{\circ} 35^{\prime} \mathrm{E}$. In Bandanyenje, safe water and sanitation coverage are poor and school children depend on perennial rivers as their water source thus exposing the majority of their population to infection. The study population comprised of 212 (105 boys and 107 girls) aged between 7-13 years, who were permanent residents of the area. Serum samples from the participants were taken and measurement of acute phase proteins and IL-6 before and after praziquantel treatment.

\section{Parasitology and blood sampling}

A school-based longitudinal intervention study was conducted and involved examination and treatment of the study population at baseline, 6 weeks and at 2 year follow up surveys. Stool and urine samples were collected from 212 schoolchildren at baseline and follow up on three consecutive days and were processed for schistosomiasis using the Kato Katz, Formol-ether concentration method as modified by Peters et al. [31] and the filtration technique Mott et al. [32] respectively. Blood samples were collected from the 212 children at baseline and 2 years post-treatment and their serum was used to determine acute phase proteins and IL-6.

\section{Praziquantel treatment}

All 212 schoolchildren were given praziquantel $(40 \mathrm{mg} / \mathrm{kg}$ body weight) at baseline regardless of the infection status. Praziquantel was administered at the same dose in all the children annually for 2 years.

\section{Acute phase proteins and IL-6 determination}

Serum levels of ferritin, fibrinogen, tissue plasminogen activator and procalcitonin, were determined by the magnetic bead-based immuno-assays using the Bio-Plex Pro ${ }^{\mathrm{mm}}$ human acute phase 4-plex immunoassay complete commercial kits. The Bio-plex manager software was used for running the assay, data acquisition and analysis. The analysis of IL-6 in the serum samples was done by sandwich ELISA.

\section{Statistical Analysis}

Data was analyzed using SPSS statistical software v16. Levels of serum levels of ferritin, fibrinogen, tissue plasminogen activator, procalcitonin and IL- 6 at baseline and 2 years post-treatment in the schoolchildren were compared using student $t$ test to determine the effect of praziquantel treatment on acute phase proteins and IL-6. The Mann-Whitney non parametric U-test was used to determine the effect of $S$. haematobium infection on the levels of ferritin, fibrinogen, tissue plasminogen activator, procalcitonin and IL- 6 . A value of $p<0.05$ was considered to indicate a significant difference in statistical analyses.

\section{Ethical Approval}

Ethical approval and clearance of the study was obtained from the Biomedical Research Ethics Committee (BREC-UKZN), approval code, BE 467/16 as well as the Medical Research Council of Zimbabwe (MRCZ), approval code, MRCZ/A/1958. The aims, objectives and procedures of the study were explained to the parents/guardians of the recruited children in the local language (Shona), when they were invited to participate. Written informed consent was obtained from all the guardians of the school children in Shona and English. Participation was voluntary and the parents/guardians had the right to withdraw their child/children at any time point from the study. Treatment was administered by trained medical personnel and the children were closely monitored. Written consent to transport participants' specimens to UKZN for acute phase protein determination was also obtained from parents/guardians.

\section{Results}

212 (105 boys and 107 girls) aged between 7-13 were recruited from Bandanyenje primary school located in a schistosomiasis endemic area and were successfully traced and re-examined at both follow-ups with complete sets of longitudinal parasitological data on $S$. haematobium infection. The overall pre-treatment prevalence of $S$. haematobium infection was $49(23.1 \%)$ with $20(19 \%)$ boys and $29(27.2 \%)$ girls as determined using the urine filtration technique (Table 1). There was an overall decline in $S$. haematobium prevalence from $23.1 \%$ at baseline to $0.47 \%$ at 6 weeks post-treatment and, $0.47 \% 2$ years post-treatment, an overall $87.1 \%$ reduction over 2 years $(\mathrm{p}<0.05)$. A total of $211(99.5 \%)$ children were egg negative after treatment.

\begin{tabular}{|r|c|c|c|}
\hline Variable & \multirow{2}{*}{ Baseline } & $\begin{array}{c}\text { 6 Weeks post } \\
\text { treatment }\end{array}$ & $\begin{array}{c}\text { 2 Years post } \\
\text { treatment }\end{array}$ \\
\cline { 1 - 2 } Prevalence & \multirow{2}{*}{$49(23.1 \%)$} & $1(0.47 \%)$ & $1(0.47 \%)$ \\
\cline { 1 - 1 } Cure rate & & $98 \%$ & $97.80 \%$ \\
\hline Boys & \multirow{2}{*}{$20(19 \%)$} & $1(0.47 \%)$ & $1(0.47 \%)$ \\
\cline { 1 - 1 } Cure rate & & $95 \%$ & $94.70 \%$ \\
\cline { 1 - 1 } Girls & \multirow{2}{*}{$29(27.1 \%)$} & 0 & 0 \\
\cline { 1 - 1 } Cure rate & & $100 \%$ & $100 \%$ \\
\hline
\end{tabular}

Table 1: Overall prevalence of $S$. haematobium infection in the study population according to gender at baseline and 2 years post praziquantel treatment.

No significant changes were observed in ferritin, tissue plasminogen activator and procalcitonin among the $S$. haematobium positive and negative children $(\mathrm{p}>0.05)$, Mann-Whitney non parametric test at baseline. Following repeated rounds of annual treatment changes occurred in the levels of the acute phase proteins though they were all insignificant with respect to infection status $\mathrm{p}>0.05$ Mann-Whitney non parametric test (Figure 3). Tissue plasminogen activator and procalcitonin levels increased slightly following treatment. In $S$. haematobium-uninfected children, levels of procalcitonin decreased following treatment though it was insignificant $(p>0.05)$ whilst the 
Citation: $\quad$ Chisango T, Vengesai A, Nhidza AF, Ndlovu B, Zhou D, et al. (2018) S. Haematobium Infection and Chemotherapy-Induced Changes in Interleukin-6 and Acute Phase Proteins Associated with Inflammation in School Children in a Schistosomiasis-Endemic Area. J Trop Dis 6: 266. doi:10.4172/2329-891X.1000266

Page 4 of 7

ferritin and tissue plasminogen activator marginally increased following treatment.

The Mann-Whitney test was also used to determine the effect of $S$. haematobium infection on the levels of acute phase proteins. $S$. haematobium-infected children had slightly higher levels of procalcitonin and tissue plasminogen activator before treatment though the difference of the two was not significant, $\mathrm{p}>0.05$ (Figure 1).

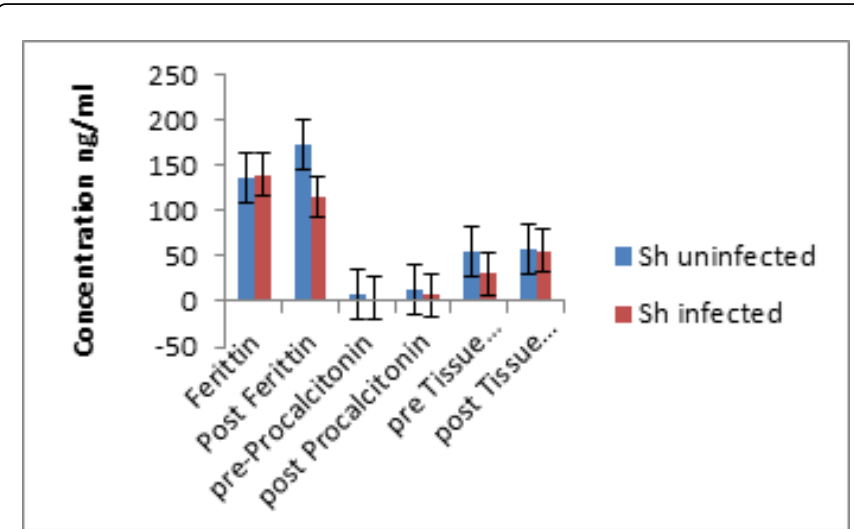

Figure 1: Mean concentration of acute phase proteins in $S$. haematobium-infected and uninfected children at baseline.

Levels of ferritin and fibrinogen were lower in $S$. haematobium positive children before treatment, however the difference was also not significant $\mathrm{p}>0.05$ (Figure 1 and Figure 2). Infection status had no bearing on the levels acute phase proteins, using the Mann-Whitney non parametric test, ferritin levels $(\mathrm{p}>0.05)$, tissue plasminogen activator $(\mathrm{p}>0.05)$, procalcitonin $(\mathrm{p}>0.05)$ were shown to be unrelated to $S$. haematobium infection. Fibrinogen levels were within normal ranges for both $S$. haematobium positive and negative children and there the Mann-Whitney test could not be performed because there was only one group (Figure 2).

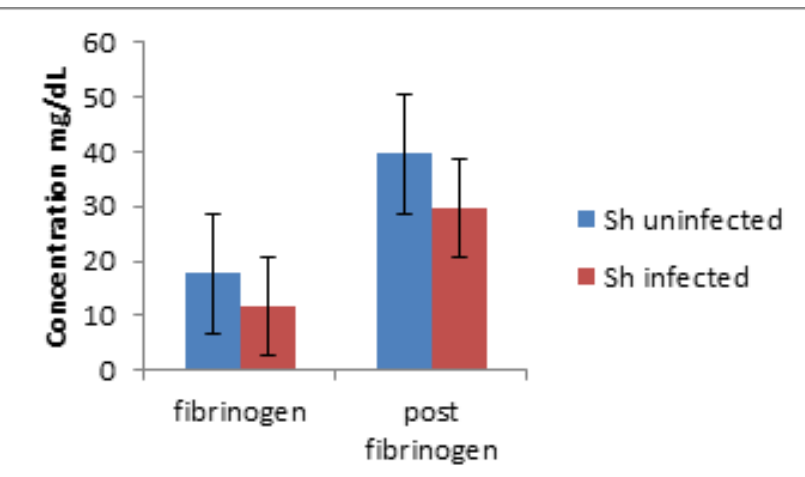

Figure 2: Mean concentration of fibrinogen in $S$. haematobium infected and uninfected children at baseline.

In order to determine the effect of treatment on the acute phase proteins the student $\mathrm{t}$ test was performed. In $S$. haematobium positive children there was a decrease in ferritin levels following treatment though it was insignificant $\mathrm{p}>0.05$, however in $S$. haematobium negative children there was a slight increase which was also no significant $(p>0.05)$. There was an insignificant increase in procalcitonin and tissue plasminogen ( $p>0.05$ and $p>0.05$ respectively) following praziquantel treatment in both $\mathrm{S}$. haematobium and positive and negative children (Figure 3).

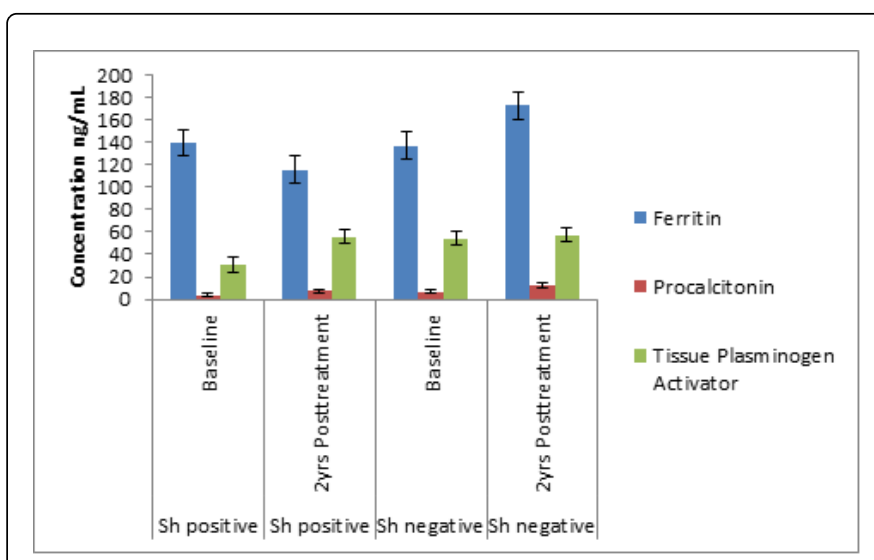

Figure 3: Mean concentration of acute phase proteins in $S$. haematobium infected and uninfected children at baseline and 2 years post praziquantel treatment.

At baseline level of IL-6 was higher in $S$. haematobium infected children than in $S$ haematobium uninfected children though the difference was insignificant $(\mathrm{p}<0.05)$ (Figure 4). Following treatment there was an increase in IL-6 in $S$. haematobium uninfected children and a decrease in IL-6 in $S$. haematobium uninfected children, however the changes were insignificant $\mathrm{p}<0.05$ in both cases. The Mann Whitney test was used to determine the effect of IL- 6 on the levels of the acute phase proteins. IL- 6 levels did not have an effect on the levels of ferritin, procalcitonin and tissue plasminogen activator $(p=0.334, p=0134$ and $p=0.847$, respectively using Mann-Whitney test). Effect of IL- 6 on fibrinogen levels could not be determined by the Mann-Whitney test because fibrinogen levels were within normal ranges for all the children.

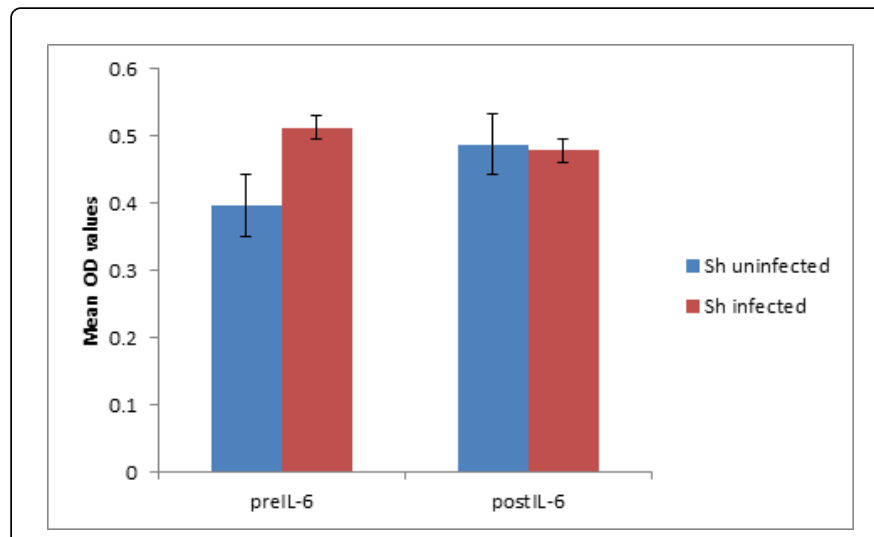

Figure 4: Mean OD values for IL 6 in $S$. haematobium infected and uninfected children before and after treatment with praziquantel.

\section{Discussion}

The $S$. haematobium prevalence was $23.1 \%$ which is slightly lower than $23.8 \%$ recorded in the province by Midzi et al. [33]. The 
prevalence of $S$. haematobium infection declined from $23.1 \%$ to $0.47 \%$ following repeated rounds of annual praziquantel treatments (Table 1). This decline in prevalence resulted in a decline in the $S$. haematobium worms laying the eggs because of worm damage caused by praziquantel. The eggs are responsible for invoking and determining the magnitude of inflammation within tissues [34]. The low levels of acute phase proteins post treatment reflect the low prevalence of infection 2 years following repeated rounds of annual praziquantel treatment. If less children are infected (less exposure of $S$. haematobium eggs in tissues), the extent of inflammation will be reduced since the tissue-trapped eggs are responsible for inflammation.

The current study is the first in reporting the relationship between fibrinogen, ferritin, procalcitonin and tissue plasminogen activator and $S$. haematobium infection in school-going children. The effect of IL-6 on the circulating four acute phase proteins is also reported for the first time in this study. This study was motivated in part by the limited information regarding relationships between biomarkers of inflammation and $S$. haematobium infection. Contrary to what was expected, we observed no association between $S$. haematobium infection status and the inflammatory markers (Figure 1); fibrinogen, ferritin, tissue plasminogen activator and procalcitonin before treatment with prazequantel. Using the Mann-Whitney non parametric test all four acute phase proteins did not show any association with $S$. haematobium infection status. Repeated exposure to inflammatory agents, common in schistosomiasis endemic settings where there is frequent exposure to cercariae infested water is expected to result in constant elevations in acute-phase proteins and other inflammatory markers. Previous studies had reported relationships between $S$. haematobium and other acute phase proteins albeit with conflicting results. It is against this background that we evaluated the levels of the four acute phase proteins. Positive associations between levels of acute phase proteins such as C-reactive protein and hepcidin and schistosoma infections have been reported [24,25]. Ferritin has also been reported to beraised in inflammatory diseases [26] but such an association was not evident in our study though in the reported case the prevalence of $S$. haeamtobium was high. Our observation can be attributed to the moderate prevalence in our study group or simply just that other markers like C-reactive protein are better markers than the markers we evaluated. The expectation that a combination of more than one measure would perform substantially better than any single one was not supported by our observations.

Serum concentration of acute phase proteins typically peak within 24 to $48 \mathrm{~h}$ after the initiation with a decline coinciding with the recovery from the infection. It has been noted that acute phase proteins decline within 4-7 days after the initial stimulus if no further stimulus occurs and that repeated exposure to the agent stimulating inflammation results in chronic inflammation with levels of acute phase proteins being continuously elevated [35]. Circulating acute phase levels are felt to be a reflection of the response to proinflammatory cytokines such as IL-6 $[15,28]$. There were no significant differences in circulating IL-6 between $S$. haematobium infected and uninfected school children and the levels in both groups were not high (Figure 4). Inflammation with T-cell activation is a distinct characteristic of $S$. haematobium infection and as such, markers of inflammation are expected to be in circulation $[36,37]$. A Th2 immune response is induced by schistosomes through Th1 down-regulation via increased IL-6 production. Host macrophages recognize the larvae of schistosomes which induces secretion of IL-6. In children who are $S$. haematobium positive induction of the Th2 response would be expected resulting in increased production of IL 6 [38].
Following treatment there were no significant changes in the level of IL-6 (Figure 4). This was unexpected especially considering the fact that studies have shown that treatment of schistosomiasis with praziquantel induces noticeable changes in cytokine levels $[39,40]$. Marked changes in cytokines such as IL-6, IL-4, IL-5 and IL-10 occur following treatment of schistosomiasis induces marked changes in cytokine levels resulting in a shift to Th2 responses which have been associated with resistance to reinfection [41]. Exploring other cytokines not included in this study (i.e., IL-4, IL-5, and IL-13) is important in understanding the immune response to multiple parasitic infections and should be integrated in future research efforts.

Our study reveals novel insights in the use of the four acute phase proteins in evaluating inflammation in $S$. haematobium, albeit with some minor limitations such as sample size and availability of resources. General limitations of assessing serum levels of inflammatory markers such as acute phase proteins and cytokines are the relatively non-specificity, short half-life, nonspecific induction, and serum levels not reflecting biologic activity. Notwithstanding these limitations, serum levels of some of these biomarkers have yielded important insights in the level of inflammation in parasitic diseases.

\section{Conclusion}

The central question in this study was whether IL-6, ferritin, fibrinogen, tissue plasminogen activator and procalcitonin are suitable as identifiers for inflammation in $S$. haematobium infection and whether their levels would change following repeated rounds of annual praziquantel treatments. Our results show no significant association between $S$. haematobium infection status and level of the four acute phase proteins ferritin, fibrinogen, tissue plasminogen activator and procalcitonin. However, marginal increases were observed in levels of procalcitonin and tissue plasminogen activator in $S$. haematobium infected children. Treatment using praziquantel did not significantly affect the levels of the four acute phase proteins. There is a need to identify and select of the most appropriate biomarkers of inflammation in children since identifying individuals who are vulnerable early stages of inflammation allows better prognosis and prevents the use of invasive, costly and time consuming procedures to determine those suffering from pathological complications of chronic inflammation conditions in future.

\section{Authors' Contribution}

TJC, BN and TM developed the field study design, acute phase proteins and IL 6 immunoassays and analyzed the data. While AV, AFN, EPS, DZ and TM conducted field and sampling work. TJC, AV, AFN and BM conducted the laboratory assays. TJC and TM conducted the initial statistical analyses. All authors contributed to the manuscript.

\section{Competing Interests}

The authors declare that they have no competing interests.

\section{Acknowledgements}

We are grateful to the University of KwaZulu-Natal (College of Health Sciences postgraduate research grant) for the financial support of this research. 
Citation: $\quad$ Chisango T, Vengesai A, Nhidza AF, Ndlovu B, Zhou D, et al. (2018) S. Haematobium Infection and Chemotherapy-Induced Changes in Interleukin-6 and Acute Phase Proteins Associated with Inflammation in School Children in a Schistosomiasis-Endemic Area. J Trop Dis 6: 266. doi:10.4172/2329-891X.1000266

Page 6 of 7

\section{Ethics Approval}

This manuscript draws from our work which received ethical approval from The Medical Research Council of Zimbabwe (MRCZ/A/ 1958) and BREC (BE467/16). All information pertaining to the discussion was obtained prior to written consent by the parents/ guardian of the children participating in the study.

\section{References}

1. King CH, Dickman K, Tisch DJ (2005) Reassessment of the cost of chronic helmintic infection: A meta-analysis of disability-related outcomes in endemic schistosomiasis. Lancet 365: 1561-1569.

2. Bustinduy AL, Thomas CL, Fiutem JJ, Parraga IM, Mungai PL, et al. (2011) Measuring fitness of Kenyan children with polyparasitic infections using the 20-meter shuttle run test as a morbidity metric. PLoS Negl Trop Dis 5: e1213.

3. Butler SE, Muok EM, Montgomery SP, Odhiambo K, Mwinzi PM, et al. (2012) Mechanism of anemia in Schistosoma mansoni-infected school children in Western Kenya. Am J Trop Med Hyg 87: 862-867.

4. Ellis MK, Li Y, Hou X, Chen H, McManus DP (2008) STNFR-II and sICAM-1 are associated with acute disease and hepatic inflammation in schistosomiasis japonica. Int J Parasitol 38: 717-723.

5. Kjetland EF, Ndhlovu PD, Gomo E, Mduluza T, Midzi N, et al. (2006) Association between genital schistosomiasis and HIV in rural Zimbabwean women. AIDS 20: 593-600.

6. Leenstra T, Coutinho HM, Acosta LP, Langdon GC, Su L, et al. (2006) Schistosoma japonicum reinfection after praziquantel treatment causes anemia associated with inflammation. Infect Immun 74: 6398-6407.

7. Downs JA, Mguta C, Kaatano GM, Mitchell KB, Bang H, et al. (2011) Urogenital schistosomiasis in women of reproductive age in Tanzania's Lake Victoria region. Am J Trop Med Hyg 84: 364-369.

8. Vodovotz Y, Constantine G, Rubin J, Csete M, Voit E, et al. (2009) Mechanistic simulations of inflammation: Current state and future prospects. Math Biosci 217: 1-10.

9. Burke ML, Jones MK, Gobert GN, Li YS, Ellis MK, et al. (2009) Immunopathogenesis of human schistosomiasis. Parasite Immunol 31: 163-176.

10. Colley DG, Secor WE (2014) Immunology of human schistosomiasis. Parasite Immunol 36: 347-357.

11. Wilson S, Vennervald BJ, Dunne DW (2011) Chronic hepatosplenomegaly in African school children: A common but neglected morbidity associated with schistosomiasis and malaria. PLoS Negl Trop Dis 5: el149.

12. Hagan P, Appleton CC, Coles GC, Kusel JR, Tchuem-Tchuente L (2004) Schistosomiasis control: Keep taking the tablets. Trends Parasitol 20: 92-97.

13. Munoz P, Valerio M, Puga D, Bouza E (2010) Parasitic infections in solid organ transplants receipients. Infec Dis Clin North Amer 24: 461-495.

14. Heegaard PM, Godson DL, Toussaint MJ, Tjonehoj K, Larsen LE, et al. (2000) The acute phase response of haptoglobin and serum amyloid A (SAA) in cattle undergoing experimental infection with bovine respiratory syncytial virus. Vet Immunopathol 77: 9-15.

15. Baumann H, Gauldie J (1990) Regulation of hepatic acute phase plasma protein genes by hepatocyte stimulating factors and other mediators of inflammation. MolBiol Med 7: 147-159.

16. Johnson HL, Chiou CC, Cho CT (1999) Applications of acute phase reactants in infectious diseases. J Microbiol Immunol Infect 32: 73-82.

17. Peracaula R, Sarrats A, Rudd PM (2010) Liver proteins as sensor of human malignancies and inflammation. Proteomics Clin Appl 4: 426-431.

18. Whicher JT, Bella M, Southall PJ (1981) Inflammation measurements in clinical management. Diagnos Med 4: 62.

19. Rashid SA, O'quigley J, Axonanda TR, Coopere H (1982) Plasma protein profiles and prognosis in gastric cancer. Br J Cancer 45: 390.
20. Shaverymuttu SH, Hodgsonh JF, Chadwick VS, Pepys MB (1986) Differing acute phase responses in Crohn's disease and ulcerative colitis. Gut 27: 809-813.

21. Gauldie J, Richards C, Harnish D, Lansdorp P, Baumann H (1987) Interferon $\beta 2 / \mathrm{B}$-cell stimulatory factor type 2 shares identity with monocyte-derived hepatocyte-stimulating factor and regulates the major acute phase protein response in liver cells. Proc Natl Acad Sci USA 84: 7251-7255.

22. Gauldie J, Lamontagne L, Stadnyk A (1985) Acute Phase Response in Infectious Disease. Surv Synth Path Res 4: 126-151.

23. Levels JH, Geurts P, Karlsson H, Maree R, Ljunggren S, et al. (2011). High-density lipoprotein proteome dynamics in human endotoxemia. Proteome Sci 9: 34.

24. Coutinho HM, Leenstra T, Acosta LP, Su L, Jarilla B, et al. (2006) Proinflammatory cytokines and c-reactive protein are associated with undernutrition in the context of Schistosoma Japonicum infection. Am J Trop Med Hyg 75: 720-726.

25. Jason J, Archibald LK, Nwanyanwu OC, Bell M, Jensen RJ, et al. (2001) The effects of iron deficiency on lymphocyte cytokine production and activation: preservation of hepatic iron but not at all cost. Clin Exp Immunol 126: 466-473.

26. Ayoya MA, Spiekermann-Brouwer GM, Stoltzfus RJ, Nemeth E, Habicht JP, et al. (2010) Alpha 1-acid glycoprotein, hepcidin C-reactive protein, and serum ferritin are correlated in anemic schoolchildren with Schistosoma haematobium. Am J Clin Nutr 91: 1784-1790.

27. Prowse KR, Baumann H (1989) Interleukin-1 and interleukin-6 stimulate acute-phase protein production in primary mouse hepatocytes. J Leukoc Biol 45: 55-61.

28. Heinrich PC, Behrmann I, Muller-Newen G, Schaper F, Graeve L (1998) Interleukin-6-type cytokine signalling through the gp130/Jak/STAT pathway. Biochem J 334: 297-314.

29. Streetz KL, Wustefeld T, Klein C, Manns MP, Trautwein C (2001) Mediators of inflammation and acute phase response in the liver. Cell Mol Biol 47: 661-673.

30. Wright EK, De Cruz P, Gearry R, Day AS, Kamm MA,(2014) Fecal biomarkers in the diagnosis and monitoring of Crohn's Disease. Inflammatory Bowel Diseases 20: 1668-1677.

31. Peters PA, El Alamy M, Warren KS, Mahmoud AA (1980) Quick Kato smear for field quantification of Schistosoma mansoni eggs. Am J Trop Med Hyg 29: 217-219.

32. Mott KE, Baltes R, Bambagha J, Baldassini B (1982) Field studies of the reusable polyamide filter for detection of $\mathrm{S}$. haematobium eggs by urine filtration. Proper nmedlizin and Parasitologie 33: 227-228.

33. Midzi N, Mduluza T, Chimbari MJ, Tshuma C, Charimari L, et al. (2014) Distribution of schistosomiasis and soil transmitted helminthiasis in Zimbabwe: Towards a National Plan of Action for Control and Elimination. PLoS Negl Trop Dis 8: e3014.

34. Jain S, Gautam V, Naseem S (2011) Acute-phase proteins: As diagnostic tool. J Pharm Bioallied Sci 3: 118-127.

35. Riet EV, Hartgers FC, Yazdanbakhsh M (2007) Chronic helminth infections induce immunomodulation: Consequences and mechanisms. Immunobiology 212: 475-490

36. Hoffmann KF, Wynn TA, Dunne DW (2002) Cytokine-mediated host responses during schistosome infections: Walking the fine line between immunological control and immunopathology. Adv Parasitol 52: 265-307.

37. La Flamme AC, MacDonald AS, Pearce EJ (2000) Role of IL-6 in directing the initial immune response to schistosome eggs. J Immunol 164: 2419-2426.

38. Grogan JL, Kremsner PG, Deelder AM, Yazdanbakhsh M (1996) Elevated proliferation and interleukin-4 release from $\mathrm{CD} 4+$ cells after chemotherapy in human Schistosoma haematobium infection. Eur J Immunol 26: 1365-1370.

39. Medhat AM, Shehata K, Bucci S, Mohamed AD, Dief S, et al. (1998) Increased interleukin- 4 and interleukin-5 production in response to 
Citation: $\quad$ Chisango T, Vengesai A, Nhidza AF, Ndlovu B, Zhou D, et al. (2018) S. Haematobium Infection and Chemotherapy-Induced Changes in Interleukin-6 and Acute Phase Proteins Associated with Inflammation in School Children in a Schistosomiasis-Endemic Area. J Trop Dis 6: 266. doi:10.4172/2329-891X.1000266

Page 7 of 7

Schistosoma haematobium adult worm antigens correlates with lack of reinfection after treatment. J Infect Dis 178: 512-519.

40. Roberts M, Butterworth AE, Gachuhi T, Ouma JH, Sturrock RF, et al. (1993) Immunity after treatment of human schistosomiasis: association between cellular responses and resistance to re-infection. Infect Immun 61: 4984-4993

41. Nixon DE, Landay AL (2010) Biomarkers of immune dysfunction in HIV. Curr Opin HIV AIDS 5: 498-503. 